\title{
The Effect of Alpha Tocopherol on Body Organs of Neonatal Rats Exposed to Vanadium
}

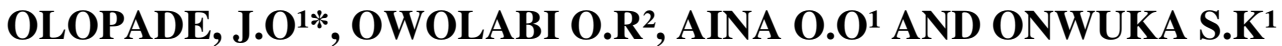

\author{
${ }^{1}$ Department of Veterinary Anatomy, University of Ibadan, Nigeria \\ ${ }^{2}$ Department of Biomedical Sciences, College of Health Science, Ladoke Akintola University of Technology, Ogbomoso, Nigeria
}

\begin{abstract}
The objective of this work was to investigate the effects of $\alpha$-Tocopherol (vitamin E) on the organs of neonatal rats exposed to Vanadium. Organ histology show that vanadium through lactation induced pathological changes including congestion and haemorrhages at the renal cortex, severe diffuse vacuolar degeneration of the hepatocytes, peribronchiolar hemorrhage, interstitial congestion and proliferation of pneumocytes. Also, the testes of the pups revealed slight reduction in germinal epithelium with the destruction of the spermatogenic cells although the interstitial connective tissue appeared relatively unaffected. The pups whose dams were treated with vanadium and $\alpha$-Tocopherol displayed morphology similar to control in these organs. In this study however, no striking distinguishing difference was observed in the heart, thymus and spleen of lactating pups exposed to vanadium when compared to controls. We conclude that $\alpha$-Tocopherol treatment of vanadium exposed mothers may also be beneficial at least in part in the protection of the organs of lactating offspring. @JASEM
\end{abstract}

Vanadium with atomic number 23 is a metallic element of the first transition series and is abundant in the environment (Aragon et al., 2005). The metal exists in oxidation state from -1 to +5 and forms polymers frequently (Clark, 1973). Vanadium is used in the manufacturing of sulphuric acid, hardening of steel, in photography, manufacturing of jet aircraft engines, pthalic anhydride, pesticides, dyes, inks, pigment and as a catalyst- in the production of several materials (Wenning and Kirsch, 1988; ATSDR, 1992; Hope, 1994). In addition vanadium has attracted research attention because of its antitumor properties (Olopade et al., 2009), potentials as a vaginal contraceptives (D' Cruz et al, 1998) and also for treatment of diabetes (Refat and El-Shazly, 2010; Hu et al, 2010). The common industrial and potential medicinal use of vanadium has made it imperative to study its toxic effects (Garcia et al., 2005). Recently, we reported that antidotal research into some aspects of vanadium toxicity remains novel and limited (Olopade and Connor, 2010); Domingo (1996) reported that the placenta represents a partial barrier to vanadium exposure while Soazo and Garcia (2007) showed that the lactation route is an effective means of inducing vanadium related neurotoxicity

Vitamin E is an important antioxidant; and the term vitamin $\mathrm{E}$ denotes several naturally occurring tocophenols and tocotrienols in which alphatocopherol is responsible for most of the activities of vitamin E (Dibier and Larondelle, 2005). Alphatocopherol has been known to improve erectile dysfunction in hypertensive rats (Ushiyama et al., 2008), and alleviate vanadium induced lipid peroxidation, and testicular pathologies in rats (Haider at al., 1991; Uche et al., 2008).

The objective of this study is to observe the protective effect of alpha tocopherol on vanadium (both administered through lactation route) induced histopathology of various body organs of neonatal rats.

*Email: jkayodeolopade@yahoo.com

\section{MATERIALS AND METHODS}

Chemical: All the materials used were of analytical grade. Sodium metavanadate was a product of Sigma Aldrich, USA. Vitamin E 1000.I.U USP was a product of Good N' Natural Manufacturing corp. Holbrook, U.S.A. Albino Wistar rats were obtained and housed in the experimental animal house of the neuroscience unit of the Department of Veterinary Anatomy of the Faculty of Veterinary Medicine, University of Ibadan, Ibadan. The animals were fed with pelleted rat feed and water ad libitum.

Methods: The neonatal rats were divided into three groups based on the treatment of their mothers.

Group A: Mothers were not treated.

Group B: Mothers were treated with $3 \mathrm{mg} / \mathrm{kg}$ b.w./day of vanadium (sodium metavanadate), IP for 14 days from first day of delivery.

Group C: Mothers were treated with $3 \mathrm{mg} / \mathrm{kg}$ b.w./day of vanadium (sodium metavanadate), IP for 14 days from first day of delivery and also $500 \mathrm{mg} / \mathrm{kg}$ b.w. of Vit. E 72 hourly in the same period

Sample Collection: The rat pups were euthanized with a combination of xylazine $(10 \mathrm{mg} / \mathrm{kg})$ and ketamine $(100 \mathrm{mg} / \mathrm{kg})$. Each organ, lungs, liver, kidney, testes, thymus, heart and spleen were dissected out, trimmed off the attached tissues and a portion fixed in $4 \%$ formalin and processed for histology. Pictures were taken using a canon digital camera.

\section{RESULTS AND DISCUSSION}

The histopathological chances that occurred from vanadium exposure in 7 organs and the protective effect of $\alpha$-Tocopherol are reported below.

Histopathology of the Kidney of lactating neonatal rat pups (PND 14) whose mothers have been exposed to $S M V(3 \mathrm{mg} / \mathrm{kg})$ from PND 1-14 (Fig 1A-C): The 
kidneys of vanadium exposed pups showed congestion and haemorrhages at the renal cortex. The congestive and haemorrhagic lesions were interstitial, multifocal and severe. The pups whose dams were treated with vanadium and vitamin $\mathrm{E}$ were either less congested or displayed morphology similar to control.

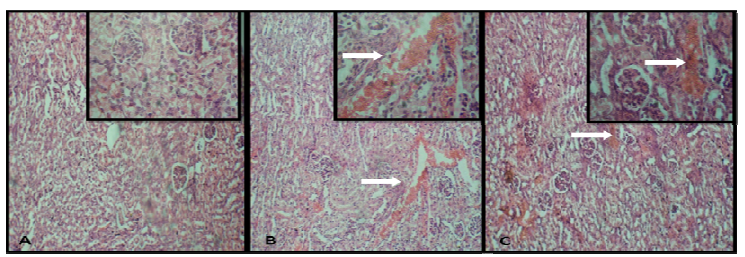

Fig 1: Histopathology of the Kidney of lactating neonatal rat pups (PND 14) whose mothers have been exposed to SMV $(3 \mathrm{mg} / \mathrm{kg})$ from PND 1-14.A: control showing no visible lesions; B (Vanadium treated) showing severe cortical and interstitial haemorhages (arrows); C(Vanadium+ Vit E) moderate cortical congestion (M x100). Insets show pictures at Mx400.

Histopathology of the Liver of lactating neonatal rat pups (PND 14) whose mothers have been exposed to SMV (3mg/kg) from PND $1-14$ (Fig 2A-C): Vanadium through lactation induced severe diffuse vacuolar degeneration of the hepatocytes. In some of the pups, there were also focally extensive areas of hepatic necrosis, individualized hepatic cells and loss of the chord arrangement. These lesions were slightly ameliorated in pups whose dams were injected with vanadium and vitamin $\mathrm{E}$.
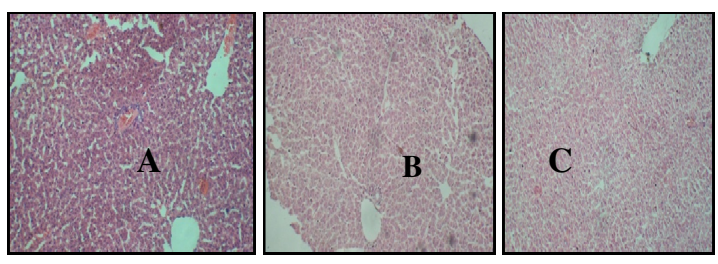

Fig 2: Histopathology of the Liver of lactating neonatal rat pups (PND 14) whose mothers have been exposed to SMV (3mg/kg) from PND 1-14. A (Control) shows normal hepatocytes and chord arrangement in livers of pups.(B\&C) Shows diffuse vacuolar degeneration of hepatocytes.(Mx100).

Histopathology of the Lungs of lactating neonatal rat pups (PND 14) whose mothers have been exposed to $S M V(3 \mathrm{mg} / \mathrm{kg})$ from PND 1-14 (Fig 3A-C): In the lungs of vanadium exposed animals, there was evidence of peribronchiolar hemorrhage, interstitial congestion and proliferation of pneumocytes. The latter probably led to the thickening of the alveoli. The lungs of pups whose dams were injected with vitamin $E$ and vanadium had a cellular morphology that was similar to those of control pups.

Histopathology of the Testes of lactating neonatal rat pups (PND 14) whose mothers have been exposed to $S M V(3 \mathrm{mg} / \mathrm{kg})$ from PND 1-14 (Fig 4 A-C): The testes of vanadium exposed pups showed slight reduction in germinal epithelium with the destruction of the spermatogenic cells although the interstitial connective tissue appeared slightly unaffected. Some tissues of vanadium treated animals however did not show any striking pathological lesions. The pups whose dams were treated with vanadium and vitamin E displayed morphology similar to control.

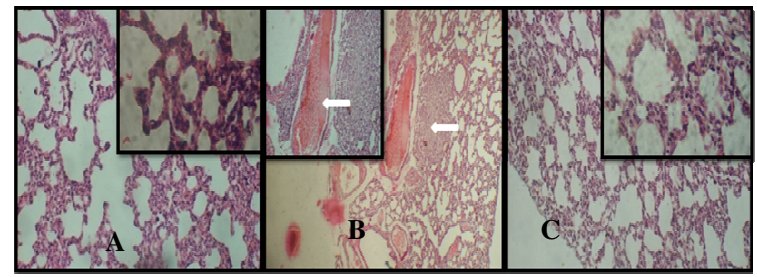

Fig 3: Histopathology of the Lungs of lactating neonatal rat pups (PND 14) whose mothers have been exposed to SMV (3mg/kg) from PND 1-14 ().A: control showing no visible lesions; B (Vanadium treated) showing severe congestion and foci of pneumocyte proliferation (arrows); C(Vanadium+ Vit E) showing no visible lesions (M x100). Insets show pictures at Mx400.

Histopathology of the Heart, Thymus, Spleen of lactating neonatal rat pups (PND 14) whose mothers have been exposed to $S M V(3 \mathrm{mg} / \mathrm{kg})$ from PND 114(Fig $5 \mathrm{~A}-\mathrm{C}$ ): No striking distinguishing difference was observed in the heart, thymus and spleen of lactating pups exposed to vanadium only and those exposed to vanadium and vitamin $\mathrm{E}$ when compared to the control. Our results show that vanadium through lactation induced pathological changes in most organs. The observations in the kidney and liver after vanadium treatment is consistent with the observations of Wei et al (1982), Domingo et al (1985), Shrivastava eta al (2007), Uche et al (2008) and Olopade et al (2009) who in their works observed one or more of corticomedullar microhaemorrhagic foci and acute renal tubular necrosis in kidneys, degenerative changes, vacuolation, fatty change, and partial cell necrosis in these organs. The toxicity caused in the lungs is particularly of epidemiological importance because vanadium is known to enter the body systems through inhalation (Avila-Costa et al., 2004). This study also shows peribronchiolar hemorrhage, interstitial congestion and proliferation of pneumocytes in the lungs; Barceloux (1999) reported nasal congestion, bronchitis and pneumonitis in man as part of the respiratory pathology of vanadium while Wei et al (1982) reported the occurrence pulmonary hemorrhage in mice. In the testis neonatal rats had a slight reduction in germinal epithelium. The effect of vanadium in this study was obviously not as pronounced like the interstitial tissue destruction and degeneration of the, seminiferous tubules reported by Olopade et al (2009), and reduction in spermatogonia, destruction of seminiferous tubules, necrosis of the testicular tissues by Uche et al (2008). Since vanadium is known to induce apoptosis by cell cycle arrest (Faure et al 1995), the fact that the rats in this experiment are neonatal rats without much spermatogenic and mitotic activities may have been a reason for reduced pathological effect of vanadium. This does not remove the fact however that chronic exposure of vanadium can affect testicular development as vanadium has been known to cause the reduction of bioavailability of the androgen to the testes by 
increasing the free radical generation (Chandra et al., 2007).

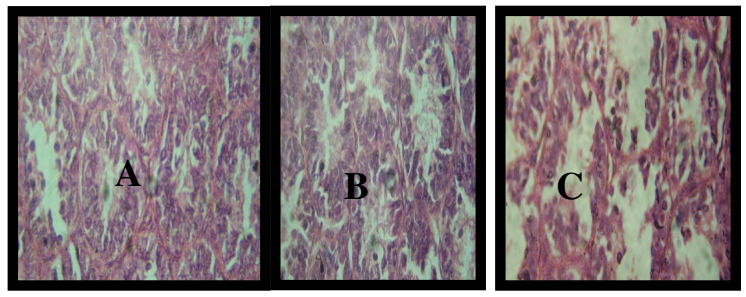

Fig 4: Histopathology of the Testes of lactating neonatal rat pups (PND 14) whose mothers have been exposed to SMV (3mg/kg) from PND 1-14. A (Control) shows normal testis with intact spermatogenic architecture and interstitial network. B (Vanadium treated) showing severe disruption and destruction of the spermatogenic cells; $\mathbf{C}$ (Vanadium+ Vit E) showing more similarity to control (M x400).

Surprisingly, we did not notice any major pathological lesions in the spleen in our neonatal rats after vanadium exposure unlike analogous reports of hypertrophy and hyperplasy in the white pulp of spleen of rats (Domingo et al 1985), and subcapsular parenchymal destruction and depletion of the lymphoid cells (Olopade et al., 2009). Though differences in the genotypic backgrounds of the cell types, such as expression of tumor suppressors or oncogenes and redox states of various cells, may act as biological determinants that allow vanadium compounds to exert cell-specific effects, more work is needed to explain the pathophysiology of vanadium toxicity in splenic tissue (Wang et al 2010; Olopade and Connor, 2010).

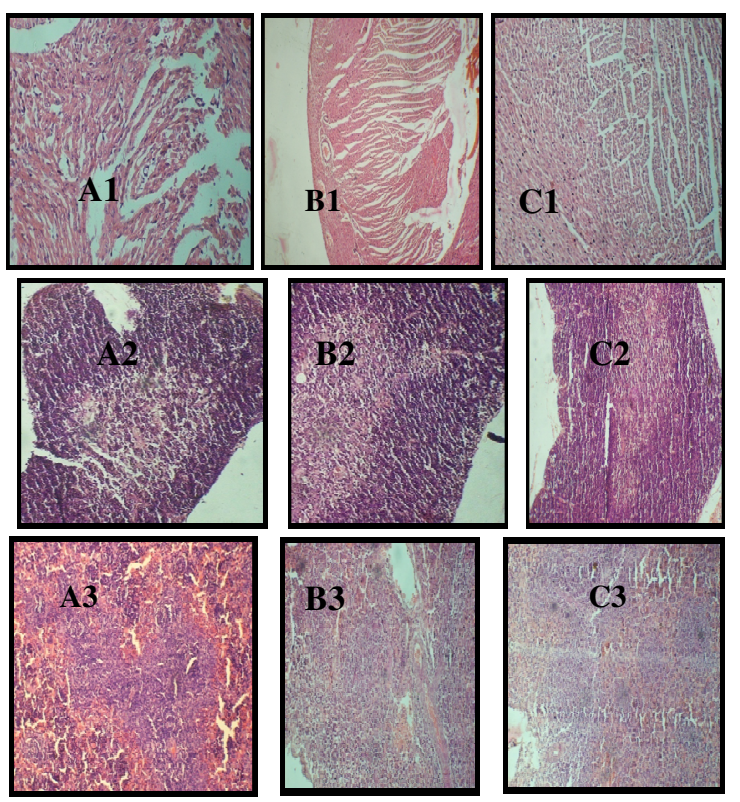

Fig 5: Histopathology of the Heart, Thymus, Spleen of lactating neonatal rat pups (PND 14) whose mothers have been exposed to SMV (3mg/kg) from PND 1-14.The heart of all groups (A1:Control,B1: Vanadium treated,C1:Vanadium+Vit E) showing no visible lesions. H\&E (M x100).The thymus of all groups (A2: Control, B2: Vanadium treated, C2: Vanadium+Vit E) showing no visible lesions. H\&E (M x100).The spleen of all groups (A3: Control, B3: Vanadium treated, C3: Vanadium+Vit E) showing no visible lesions. H\&E (M x100).

Vanadium has been known to exert most of it toxic effects through the generation of reactive oxygen species making vanadium to act as a stressor metal with oxidative stress the major mechanism of health deterioration (Chandra et al., 2007). It is thus logical that potent antioxidants be screened to assess their potent effects on vanadium induced toxicity. Various workers (Haider et al., 1991; Shrisvastava et al., 2007; Chandra et al., 2007; Uche et al., 2008) have reported the protective effect of vitamin $\mathrm{E}$ either singly or in combination with other agents against vanadium induced toxicity. Recently we observed that vitamin $\mathrm{E}$ administration through lactation as done in this work ameliorated vanadium induced (also through lactation) astrogliosis and behavioural deficits in neonatal rats (Olopade et al., submitted manuscript).

Our work has shown that vanadium toxicity in lactating mothers invariably depicts that the same condition will be transmitted to the offsprings. This is a source of concern in traditional villages like in the Nigerian Niger Delta where burning of vanadium containing crude is on the rise. Toxic exposure of lactating women who go into the ecosystem in support of their families means a transfer to their offspring who though may be kept safe at home. Our work however also shows that vitamin E treatment of the exposed mothers however may also be beneficial at least in part in the protection of the offspring

Acknowledgement: The authors acknowledge the assistance of Dr Fatola and the technical support of Messrs Ramoni, Ihekanwa, Ajayi, Mrs Adeyemo and Miss Onifade, all of Department of Veterinary of Anatomy. This work was done in part with University of Ibadan Senate Research grants to JOO

\section{REFERENCES}

Aragon, M.A; Ayala, M.E; Fortoul, T.I; Bizarro, P; Altamirano-Lozano, M (2005). Vanadium induced ultrastructural changes and apoptosis in male germ cells. Reproductive Toxicology, 20(1):127-134.

ATSDR (1992) Toxicological profile for vanadium. U.S Department of Health and Human services, Public Health service, Agency for Toxic substances and Disease Registry, Atlanta, Georgia.

Avila-Costa MR, Montiel Flores E, Colin-Barenque L, et al. 2004. Nigrostriatal modifications after vanadium inhalation: An immunocytochemical and cytological approach. Neurochemistry Research 29(7):13651369.

Barceloux, D.G (1999). Vanadium. Journal of Toxicology and Clinical Toxicology, 37: 265-278.

Chandra, A.K; Ghosh, R; Chatterjee, A; Sarkar, M (2007). Amelioration of vanadium-induced testicular toxicity and adrenocortical hyperactivity by vitamin E acetate in rats. Molecular and Cellular Biochemistry, 306(12):189-200.

Clark, R.J.H. (1973) Vanadium. In: A.F. TrotmanDickenson, Editor, Comprehensive Inorganic 
Chemistry Vol. 3, Pergamon, New York, N.Y (1973), pp. 519-521

D' cruz, O.D; Ghosh, P; Uckum, F.M. (1998). Spermicidal activity of metallocene complexes containing vanadium (IV) in humans. Biology of Reproduction, 58:1515-1526.

Debier, C; Larondelle, Y. (2005). Vitamin A and E: metabolism role and transfer to offspring. British Journal of Nutrition, 93(2): 153-174.

Domingo, J.L.( 1996). Vanadium: A review of the reproductive and developmental toxicity. Reproductive Toxicology, 10(3):175-182.

Domingo, J.L; Llobet, J.M; Tomas, J.M (1985).Short-term toxicity studies of vanadium in rats. Journal of Appllied Toxicology, 5(6):418-420.

Faure, R; Vincent, M; Dufour, M; Shaver, A; Posner, B.I (1995). Arrest of the G2/M transition of the cell cycle by protein tyrosine phosphatase inhibition: studies on a neuronal and a glial cell line. Journal of Cellular Biochemistry. 40: 389-401.

Garcia,G.B; Bian Cardi, M.E; Quirojo, A.D (2005). Vanadium (V) - Induced Neurotoxicity in the Rat Central Nervous System: A Histoimmunohistochemical study. Drug Chemical Toxicology, 28(3):329-344.

Haider, S.S; el-Eakhri, M (1991). Action of alpha tocopherol on vanadium stimulated lipid peroxidation in rat brain. Neurotoxicology, 12(1): 79-85

Hope, B.K (1994).A Global Biogeochemical Budget for Vanadium. Science of the Total Environment. 141: 110.

Hu, R; He, C; Liu, J; Wu, Y; Li, J; Feng, Z; Huang, J; Xi, $\mathrm{XG}$; Wu, Z (2010). Effects of insulin-mimeticvanadyl-poly(gamma-glutamic acid) complex on diabetic rat model. J Pharm Sci. 99(7):3041-7.

Olopade, J.O; Madhankumar, A.B; Das, A; Todorich, B; Liu, X; Slagle-Webb, B; Liang, J.J; Connor, J.R (2009. Vanadium a possible chemotherapeutic agent against astrocytoma: Proceedings of the $100^{\text {th }}$ annual meeting of the American Association of Cancer Research, Denver Colorado April 18-22, 2009, 50:1344.
Olopade, J.O; Connor J.R (2010).Vanadium and Neurotoxicity: A Review. Current Topics in Toxicology (In Press).

Refat, M.S; El-Shazly S.A (2010).Identification of a new anti-diabetic agent by combining VOSO4 and vitamin $\mathrm{E}$ in a single molecule: studies on its spectral, thermal and pharmacological properties.European Journal Medical Chemistry. 45(7):3070-9

Shrivastava S, Jadon A, Shukla S (2007). Effect of tiron and its combination with nutritional supplements against vanadium intoxication in female albino rats. Journal of Toxicological Science 32(2):185-192.

Soazo,M; Garcia, G.B (2007). Vanadium exposure through lactation produces behavioural alterations and CNS myelin deficit in neonatal rats. Neurotoxicology and Teratology, 29: 503- 510

Todorich, B; Olopade, J.O; Surguladze, N; Zhang, X; Neely, E; Connor, J (2009). The mechanism of vanadium mediated Developmental Hypomyelination is related to destruction of oligodendrocyte projenitor throudgh a relationship with ferritin and iron. Journal of Neurotoxicity Research (In Press).

Uche,F.I;Obianime,A.W;Gogo-Abite, M (2008) . Effects of Vanadium pentoxide on the Histological and Sperm parameters of male Guinea Pigs. Journal of Appllied Science and Environmental Management 12(3):107115.

Ushiyama, M; Kuramochi, T; Yagis; Kutayama, S (2008). Antioxidant treatment with alpha- tocopherol improves erectile function in hypertensives rats. Hypertension Research, 31 (5): 1007-1013.

Wang, Q; Liu, T.T; Fu, Y; Wang, K; Yang, XG (2010).Vanadium compounds discriminate hepatoma and normal hepatic cells by differential regulation of reactive oxygen species, Journal of Biological Inorganic Chemistry. 215(7):1087-97

Wei, C.I; Al Bayati, M.A; Culbertson, M.R; Rosenblatt, L.S; Hansen, L.D (1982). Acute toxicity of ammonium metavanadate in mice. Journal of Toxicology and Environmental. Health 1982, 10, 673687.

Wenning, R; Kirsch, N (1988). Vanadium. In: Handbook on Toxicity of Inorganic compounds. Marcel Dekker, New York. Eds : Seiler, H.G; Sigel, H; Sigel, A. pp.749-765. 